\title{
Can imperfect host discrimination explain partial patch exploitation in parasitoids?
}

\author{
Y. OUTREMAN ${ }^{1}$ A. LE RALEC, ${ }^{1}$ E. W A J N B E R G ${ }^{2}$ and \\ J . S . P I E R R E ${ }^{3}{ }^{1}$ E.N.S.A.R., Laboratoire d'Ecologie et Sciences Phytosanitaires, Rennes, ${ }^{2}$ I.N.R.A., Laboratoire \\ d'Ecologie des Parasitoïdes, Antibes and ${ }^{3}$ Université de Rennes I, U.M.R. 6552, Rennes, France
}

\begin{abstract}
Host discrimination by Aphidius rhopalosiphi (De Stefani Perez) (Hymenoptera: Braconidae) was first studied on the grain aphid Sitobion avenae (Fabricius) (Homoptera: Aphididae). Females tended to avoid oviposition in hosts parasitised $3 \mathrm{~h}$ earlier. No evidence of host discrimination ability on freshly parasitised hosts was suggested, however, and ovipositional experience had no effect on host discrimination.

2. The effects of host discrimination ability on the exploitation strategy of patches containing different proportions of unparasitised hosts and hosts parasitised for $3 \mathrm{~h}$ were studied. Females spent less time on patches with a higher proportion of parasitised hosts, reflecting the females' ability to perceive the potential profitability of the patch. This ability may be based on the nature of the hosts encountered (unparasitised or parasitised).

3. Incomplete exploitation of unparasitised hosts was also observed. It seems that this partial exploitation is related to the inability of A. rhopalosiphi to recognise freshly parasitised hosts. As a female may experience a risk of self-superparasitism during patch depletion, this could promote early departure from incompletely exploited patches.

4. The effect of previous experience on the patch exploitation strategy was also assessed. Females were tested twice on two patches of the same quality. Results suggested that the experience acquired during a previous visit led the females to leave the patch sooner and to lay fewer eggs in parasitised hosts.

5. Patch exploitation strategy may therefore be the result of different factors such as host discrimination and experience. The evolutionary consequences of the results are discussed.
\end{abstract}

Key words. Aphidius rhopalosiphi, experience, host discrimination, parasitoids, patch exploitation strategy, patch leaving rules.

\section{Introduction}

The question of how to use a patch optimally is a classic problem in optimal foraging theory. In parasitoids, there is a direct link between the patch exploitation strategy and the rate of progeny production. Such a relationship suggests that patch departure rules have been promoted by natural selection in a way that optimises a function linked directly to reproductive success. A number of mechanistic models has been proposed

Correspondence: A. Le Ralec, Ecole Nationale Supérieure Agronomique de Rennes, Laboratoire d'Ecologie et Sciences Phytosanitaires, 65 rue de Saint-Brieuc, 35042 Rennes Cedex, France. E-mail: leralec@roazhon.inra.fr that describe the departure of parasitoids from a patch. Among these different patch leaving mechanisms, the most well known is the model proposed by Waage (1979), based on the moth parasitoid Venturia canescens (Gravenhorst) (Hymenoptera: Ichneumonidae). Waage's mechanism suggests that females enter a patch with an initial tendency to remain on it (this is determined by the concentration of kairomones, which is related to host density). This tendency is assumed to decrease with time down to a specific threshold, at which the patch is abandoned. Each oviposition adds an increment to the current level of motivation and thereby increases the patch residence time. According to this model, the time spent on a patch should be influenced by host density. In some cases, however, each oviposition may decrease the tendency to 
remain on a patch. Such a decremental effect, termed a countdown mechanism, has been described for Venturia canescens (Driessen etal., 1995) and in an aphid parasitoid Aphidius colemani (Viereck) (Hymenoptera: Braconidae) (van Steenis etal., 1996). This mechanism converges to the fixed number rule when hosts are distributed uniformly in the environment (Iwasa et al., 1981). Finally, other authors have integrated in these mechanistic models the effect of encounters with parasitised hosts (van Lenteren, 1981; van Alphen \& Vet, 1986; van Alphen, 1993; Hemerik et al., 1993), suggesting that such encounters should have a decremental effect on patch residence time. The decremental effect associated with an encounter with parasitised hosts has been discussed by van Alphen (1993) and Wajnberg et al. (1999).

In the models discussed above, it is assumed that environmental factors influence patch residence time, but other factors may also affect the decision to leave a patch. Patch time allocation is not necessarily fixed but may vary in response to the female's physiological state (e.g. Collins \& Dixon, 1986; Rosenheim \& Rosen, 1991) or genotype (e.g. Wajnberg etal., 1999). In addition, the quest for factors inducing variability in foraging behaviour has focused largely on the effect of learning (Vet et al., 1990). Learning through experience may affect patch residence time (Papaj et al., 1994; Vet etal., 1995; Vos etal., 1998). Some authors have suggested that learning is a powerful way to optimise behaviour in an unpredictable environment (e.g. Stephens, 1990). Specifically, McNamara and Houston (1980, 1985) suggested that patch leaving rules are determined by a Bayesian-like sampling procedure: a foraging animal may have an a priori evaluation of patch profitability that is re-adjusted a posteriori through information acquired during foraging. Hence, if a parasitoid female has imperfect knowledge of patch profitability, experience may be an efficient mechanism for the optimisation of patch leaving decisions. It is not always evident, however, whether experience increases or decreases the female's tendency to leave (Vos et al., 1998).

Finally, another factor that may induce variability in patch residence time is the ability of a parasitoid female to discriminate between unparasitised and parasitised hosts. All the models described above were built for species that are assumed to discriminate their host perfectly and thus did not take into account the possibility of imperfect host discrimination. Rosenheim and Mangel (1994) introduced the problem of imperfect host discrimination in a model based on patch leaving rules. Indeed, an occasional lack in host discrimination ability was observed in a few species. During this occasional lack, the rate of superparasitism is assumed to be greater than in species with perfect host discrimination: a female may indeed re-encounter hosts that it has already parasitised and may lay an additional egg in these hosts. Rosenheim and Mangel (1994) demonstrated, with a dynamic state variable model based on the egg parasitoid Anagrus delicatus (Dozier) (Hymenoptera: Mymaridae), that, in such a case, the cost of self-superparasitism is sufficient to promote early departure from incompletely exploited patches. Nevertheless, there is no experimental evidence to support this hypothesis.
The aim of the work reported here was to investigate the effect of host discrimination ability and previous experience on the patch exploitation strategy by the aphid parasitoid Aphidius rhopalosiphi (De Stefani Perez) (Hymenoptera: Braconidae) attacking patches of one of its hosts, the grain aphid Sitobion avenae (Fabricius) (Homoptera: Aphididae). Aphidius rhopalosiphi has an important role in regulating aphid populations on cereal crops in Europe (Krespi et al., 1994). Because it has been reported that $A$. rhopalosiphi may not have the ability to discriminate between unparasitised and parasitised aphids (Gardner et al., 1984), the host discrimination ability in A. rhopalosiphi was assessed first. The effects of host discrimination ability on patch exploitation strategy and on egg distributions within hosts on patches of different qualities were then examined. Indeed, the possible lack of host discrimination in A. rhopalosiphi would make this Aphidiinae well adapted to test the patch leaving rules model of Rosenheim and Mangel (1994). Finally, the effect of previous patch experience on the decision to parasitise a host and leave a patch was assessed. Even if host discrimination is imperfect, it is possible that the experience acquired in a previous patch might lead the female to behave in a more optimal way in subsequently visited patches.

\section{Materials and methods}

\section{Insects}

The strain of Aphidius rhopalosiphi (determined by P. Starý, Institute of Entomology, Ceske Budejovice, Czech Republic) was collected in June 1996 near Rennes, France and reared in the laboratory on a mixed-age colony of the grain aphid Sitobion avenae, feeding on winter wheat Triticum aestivum cv. 'Arminda'. Hosts originated from one parthenogenetic female collected in 1990 in the same area. Colonies of both $A$. rhopalosiphi and $S$. avenae were reared in the laboratory under the following conditions: $20 \pm 1{ }^{\circ} \mathrm{C}, 70 \pm 10 \% \mathrm{RH}, \mathrm{LD} 16: 8 \mathrm{~h}$. For the experiments, only third-instar nymphs of $S$. avenae were used as hosts. Parasitoid females were isolated from mummies and, after emergence, enclosed in plastic tubes $(22 \times 1 \mathrm{~cm})$ with moistened cotton, a diluted drop of honey, and one male for mating. Only mated 1-2-day-old females were used in experiments.

\section{Host discrimination in Aphidius rhopalosiphi}

Single parasitoid females were placed with 10 aphids in a 4-cm diameter Petri dish, which was considered to be a patch. Each host of the patch was placed alone on a piece of wheat leaf. To assess the ability of $A$. rhopalosiphi to discriminate between parasitised and unparasitised aphids, observations were made on parasitoids exposed to patches containing varying proportions of already parasitised hosts: 0,50 , or $100 \%$. In patches containing $50 \%$ of hosts already parasitised, unparasitised aphids were distinguishable from parasitised aphids by the shape of the piece of leaf on which they were 
placed. Parasitised aphids used in the experiment had been attacked $3 \mathrm{~h}$ prior to the trial by a conspecific female. The behaviour of 10 inexperienced females (no ovipositional experience) and 10 experienced females (ovipositional experience) was compared on these different patches. Experienced females were individual wasps that had been placed on a leaf containing 10 unparasitised hosts for $1 \mathrm{~h}, 3 \mathrm{~h}$ prior to the trial. Only females that had oviposited at least once in hosts were used for the experiment.

The oviposition behaviour of each female was observed continuously for $1 \mathrm{~h}$ under a binocular microscope. Only one attack per aphid was allowed. A host was considered to have been attacked when the female had stung it then departed from it. Subsequent encounter with the attacked host was excluded. Attacked aphids were removed immediately and replaced with an aphid of the same initial status (healthy or parasitised) in order to keep the patch quality constant. Replacing an aphid took only $1-2 \mathrm{~s}$ and did not affect the parasitoid behaviour. All attacked aphids were caged individually on wheat. After 3 days, attacked aphids were dissected in a droplet of insect saline solution and the number of parasitoid larvae was counted. Analysis was based on the status of aphids that had been attacked and the presence or absence of parasitoid larva(e) within the hosts after attack.

In order to estimate the host discrimination ability in $A$. rhopalosiphi, a binomial non-parametric test (exact binomial test) was devised. The null hypothesis $H_{0}$ was that $A$. rhopalosiphi does not have the ability to discriminate between parasitised and unparasitised hosts. In the patch with $50 \%$ of the hosts already parasitised, females had to choose between parasitised and healthy aphids, both of which would have the same probability of being encountered. Under such conditions, let $n_{j}$ be the number of already parasitised aphids attacked by the $j$ th female among $M_{j}$, the total number of hosts attacked by the $j$ th female. Under $H_{0}$, the distribution of the probabilities of obtaining $n_{j}$ attacked parasitised aphids follows a binomial distribution $B\left(M_{j} ; P\right)$ with $P=0.5$. In the present test, the $j$ th female was considered to be able to recognise parasitised hosts if $P\left(X \leqslant n_{j}\right)<\alpha$ (with $\alpha=0.05$ ), where $P\left(X \leqslant n_{j}\right)$ is the probability for the $j$ th female of attacking fewer than $n_{j}$ already parasitised aphids. According to this test, $m$ was the total number of females that was considered to be able to recognise parasitised hosts, among $N$, the total number of tested females. Under $H_{0}, m$ follows a binomial distribution $B(N ; \alpha)$. The test consists of calculating $P(Y \geqslant m)$, which represents the probability of obtaining a greater number of females considered as being able to recognise parasitised hosts over all tested females. If $P(Y \geqslant m)<0.05, H_{0}$ is rejected and A. rhopalosiphi is considered to be able to discriminate between healthy and parasitised hosts.

\section{Effects of host discrimination and experience on patch exploitation strategy}

Experiments were carried out in a 9-cm diameter Petri dish placed in the centre of a $16-\mathrm{cm}$ diameter glass box. A wheat leaf $(8 \mathrm{~cm}$ long) infested with 12 aphids was placed in the Petri dish and considered to be a patch. To assess the effect of host discrimination on patch exploitation strategy, observations were carried out with patches containing various proportions of already parasitised hosts: $0,33,67$, or $100 \%$. Each was replicated 10 times and only females that had previous ovipositional experience were used. As for the first experiment, already parasitised aphids had been attacked $3 \mathrm{~h}$ prior to the trial by a conspecific female. The initial status of the aphid was marked by amputation of the distal portion of one antenna (Mackauer, 1972). This marking was alternated at each replicate between the two kinds of aphids throughout the experiment.

At the beginning of the experiment, a female was introduced into the Petri dish. The trial started when the female was on the leaf (i.e. on the patch) searching for hosts. The time duration of the following behaviours was recorded with an accuracy of $0.5 \mathrm{~s}$ : off the patch, on the patch, standing still, preening, and handling hosts. The total number of host encounters was also recorded. A host was considered to be encountered when the female stopped its searching activity after both location and contact with that host. During an encounter, the parasitoid displayed antennal tapping and/or stabbing behaviour. Piercing the skin of the encountered aphid with the ovipositor was interpreted as an attack. Any host encounter that did not lead to a stabbing behaviour was considered to be a so-called host antennal rejection, which was also recorded. The entire sequence, from the time to locate an aphid until resumption of searching, was considered as the handling time of that aphid. During patch exploitation, the hosts were not replaced (i.e. the patches may suffer a continuous depletion).

An experiment was terminated when the parasitoid left the patch either by flight or by walking from the Petri dish into the 16-cm diameter glass box. On some occasions, though, wasps walked a few millimetres off the patch before turning and resuming searching on the patch. Outside the patch, A. rhopalosiphi never probed or drummed. So, a complete patch visit consisted of a number of on-patch and off-patch bouts. The occurrence of short excursions outside the patch entails the problem of how to define the end of a patch visit. An arbitrary criterion is then commonly used in studies of patch time allocation by parasitic wasps (Wajnberg etal., 1999). In the present study, a trial was also stopped when the female was off the patch for more than $2 \mathrm{~min}$. To check whether this arbitrary criterion had any artefactual effect on the results, all computations were also performed with a threshold value of $60 \mathrm{~s}$ and $30 \mathrm{~s}$. Results led to the same conclusion qualitatively in all cases.

Total patch time was defined as the total time elapsed between entering the patch for the first time and the moment the female left the patch. All excursions outside the patch were deducted. Total patch time includes both time for active search and time for non-searching behaviour such as standing still, preening, and handling hosts. After analysing the behavioural records, it appeared that time devoted to preening and standing still was $<4 \%$ of the total patch time. These behaviours were therefore neglected and search time was set as the total patch time minus the total handling time. 
(a) $100 \%$ unparasitised aphids

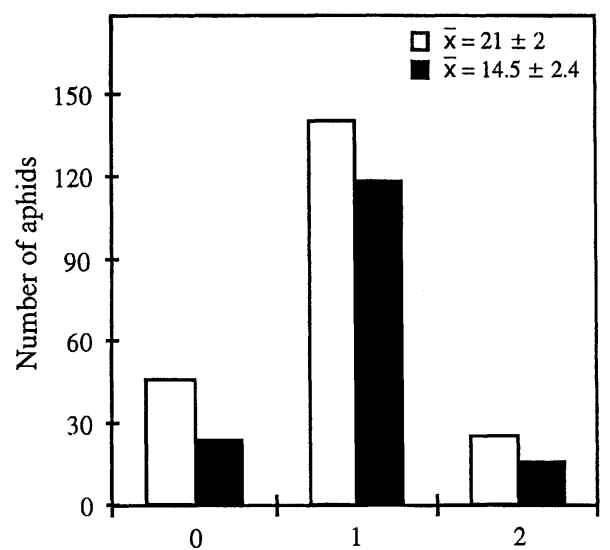

Number of parasitoid larvae (b) $100 \%$ parasitised aphids

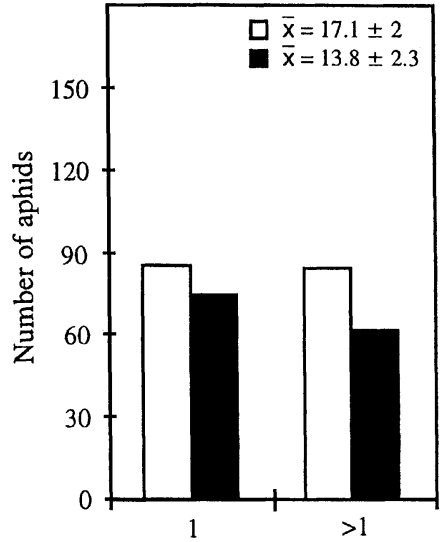

Number of parasitoid larvae

(c) $50 \%$ unparasitised aphids $+50 \%$ parasitised aphids

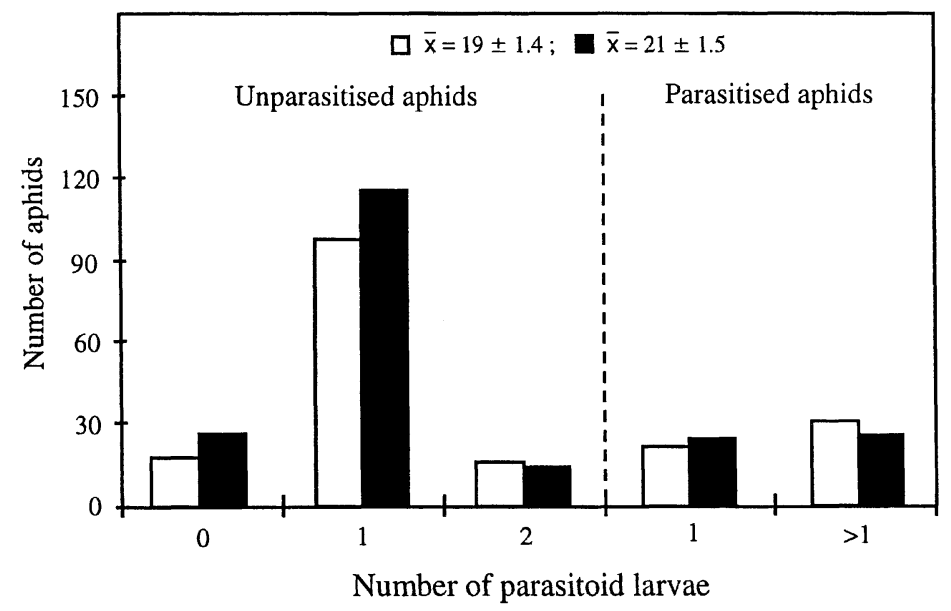

Inexperienced females

Experienced females

Fig. 1. Distribution of the number of parasitic larvae found inside once-attacked grain aphids for two groups of females of Aphidius rhopalosiphi (inexperienced and experienced females) on patches containing a different proportion of parasitised aphids: (a) 100\% unparasitised aphids, (b) $100 \%$ parasitised aphids, (c) $50 \%$ unparasitised aphids $+50 \%$ parasitised aphids. The mean number of aphids attacked by individual parasitoids for each treatment is indicated by $\bar{x} \pm$ SE. All replicates were grouped for each treatment.

To assess the role of previous patch experience on patch exploitation behaviour, the female was removed immediately at the end of the trial, kept with no hosts for $3 \mathrm{~h}$, then left to settle on a second patch of the same quality. The two successive trials are designated as series 1 and series 2 . All behaviours recorded in series 1 were also recorded in series 2 .

At the end of the experiment, females were dissected to check egg load because egg depletion could trigger patch departure (van Alphen \& Galis, 1983). These dissections indicated that there were still about 30 mature eggs in ovaries after departure from the second patch, so the effect of egg-load depletion was ignored. After 3 days, all aphids of each visited patch were dissected and the presence/absence and number of parasitic larvae were recorded.

For statistical analysis, data from all females were grouped within an experiment. Each recorded variable (total patch time, searching time, total number of encounters, number of antennal rejections, number of eggs laid) was tested against (1) two fixed effects, i.e. patch quality and previous experience (series 1 vs. series 2) and their interaction, and (2) the random effect, i.e. the tested female. A correlation was found between the total number of encounters and the time females spent searching in the patch (Pearson correlation coefficient $=0.48$, $P<0.001, n=80)$. Because this correlation coefficient suggests 
Table 1. Generalised linear models on the effects of patch quality, female, and previous experience on the patch exploitation strategy of Aphidius rhopalosiphi on patches containing the grain aphid Sitobion avenae as hosts.

\begin{tabular}{|c|c|c|c|c|c|c|c|c|c|c|c|}
\hline \multirow[b]{3}{*}{ Source } & \multirow[b]{3}{*}{ d.f. } & \multicolumn{10}{|c|}{ Variables (distribution family) } \\
\hline & & \multicolumn{2}{|c|}{$\begin{array}{l}\text { Patch time } \\
\text { (gamma) }\end{array}$} & \multicolumn{2}{|c|}{$\begin{array}{l}\text { Searching time } \\
\text { (gamma) }\end{array}$} & \multicolumn{2}{|c|}{$\begin{array}{l}\text { Number of encounters } \\
\text { (Poisson) }\end{array}$} & \multicolumn{2}{|c|}{$\begin{array}{l}\text { Number of antennal } \\
\text { rejections (Poisson) }\end{array}$} & \multicolumn{2}{|c|}{$\begin{array}{l}\text { Number of eggs laid } \\
\text { (Poisson) }\end{array}$} \\
\hline & & $F$ & $P$-value & $F$ & $P$-value & $F$ & $P$-value & $F$ & $P$-value & $F$ & $P$-value \\
\hline Patch quality & 3 & 44.02 & $<0.001$ & 9.61 & $<0.001$ & 5.65 & $<0.01$ & 3.08 & $<0.05$ & 12.65 & $<0.001$ \\
\hline Female & 39 & 0.69 & NS & 1.12 & NS & 3.11 & $<0.001$ & 2.94 & $<0.001$ & 0.13 & NS \\
\hline Previous experience & 1 & 20.48 & $<0.001$ & 20.43 & $<0.001$ & 5.04 & $<0.05$ & 6.46 & $<0.05$ & 6.49 & $<0.05$ \\
\hline $\begin{array}{l}\text { Interaction of patch quality } \\
\times \text { previous experience }\end{array}$ & 3 & 4.98 & $<0.05$ & 4.29 & $<0.05$ & 3.73 & $<0.05$ & 1.42 & NS & 0.12 & NS \\
\hline Error & 33 & & & & & & & & & & \\
\hline Total & 79 & & & & & & & & & & \\
\hline
\end{tabular}

that the effects of both variables are not completely confounded, these were studied separately. Analysis was carried out by means of generalised linear models assuming either a gamma or a Poisson error according to the recorded variable and a link function (McCullagh \& Nelder, 1989), using the S-plus ${ }^{\circledR}$ statistical software (MathSoft, Cambridge, Massachusetts).

\section{Results}

\section{Host discrimination in Aphidius rhopalosiphi}

In patches with only unparasitised aphids (Fig. 1a), 65-75\% were found parasitised with one larva. For both inexperienced and experienced females, 15-20\% of the attacked aphids contained no parasitoid larvae and $\approx 11 \%$ of attacked aphids contained two parasitoid larvae. Hence, cases of superparasitism were observed although only one attack per host was allowed. Actually, observations revealed that a single attack could consist of multiple stabbing. Parasitoids tried to oviposit several times when the aphid attempted to defend itself or the female misjudged the angle or position of the host. Such attacks could probably result in successive ovipositions, leading to the observed self-superparasitism. No significant difference between experienced and inexperienced females was found in the distributions of eggs over the initially unparasitised hosts $\left(\chi^{2}=3.60\right.$, d.f. $\left.=2, P=\mathrm{NS}\right)$. In patches where females were offered only previously parasitised aphids (Fig. 1b), $\approx 50 \%$ of attacked aphids contained more than one parasitoid larva. This result, which supposes some cases of conspecific-superparasitism, was not affected by ovipositional experience $\left(\chi^{2}=0.59\right.$, d.f. $\left.=1, P=\mathrm{NS}\right)$.

Finally, in the patches with $50 \%$ of the hosts already attacked (Fig. 1c), the distribution of eggs over healthy aphids was similar to the distribution of eggs over unparasitised aphids in the first type of patches (Fig. 1a) (inexperienced females: $\chi^{2}=3.34$, d.f. $=2, P=\mathrm{NS}$; experienced females: $\chi^{2}=0.48$, d.f. $=2, P=\mathrm{NS}$ ). Moreover, the distribution of eggs over parasitised aphids (Fig. 1c) did not differ from the distribution of eggs observed in the patch with only parasitised aphids (Fig. 1b) (inexperienced females: $\chi^{2}=1.25$, d.f. $=1$, $P=\mathrm{NS}$; experienced females: $\chi^{2}=0.50$, d.f. $=1, P=\mathrm{NS}$ ). Ovipositional experience did not affect these distributions of eggs over either unparasitised $\left(\chi^{2}=1.29\right.$, d.f. $\left.=2, P=\mathrm{NS}\right)$ or parasitised $\left(\chi^{2}=0.59\right.$, d.f. $\left.=1, P=\mathrm{NS}\right)$ aphids.

In patches with $50 \%$ of hosts already parasitised, both inexperienced and experienced females attacked unparasitised aphids more than parasitised hosts (Fig. 1c), suggesting that $A$. rhopalosiphi was able to recognise parasitised aphids. The binomial test described above was used to test this assumption. Highly significant tests for inexperienced $[P(Y \geqslant m)=$ $\left.8.1910^{-08}\right]$ and experienced $\left[P(Y \geqslant m)=1.610^{-09}\right]$ females were found, indicating that females were able to recognise aphids parasitised $3 \mathrm{~h}$ earlier.

In addition, ovipositional experience had no effect on host discrimination ability because no difference was found between experienced and inexperienced females in the number of previously parasitised aphids attacked $\left(\chi^{2}=0.85\right.$, d.f. $=1$, $P=\mathrm{NS})$.

\section{Effects of host discrimination and experience on patch exploitation strategy}

The patch quality had a significant effect on total patch time (Table 1). On the first patches visited (series 1), the total time spent on a patch decreased with increases in the proportion of previously parasitised aphids in that patch (Fig. 2a). On patches with only healthy aphids, the total patch time was about $17 \mathrm{~min}$ and this was reduced by half on patches with only previously parasitised aphids. There was also a significant effect of previous experience and of the interaction of patch quality $\times$ previous experience on the total patch time on the second patch of the same quality. On average, females spent significantly less time on the second patch (series 2) than on the first patch (series 1). In low-quality patches, previous experience did not induce a reduction in patch time (Fig. 2a). The patch quality 

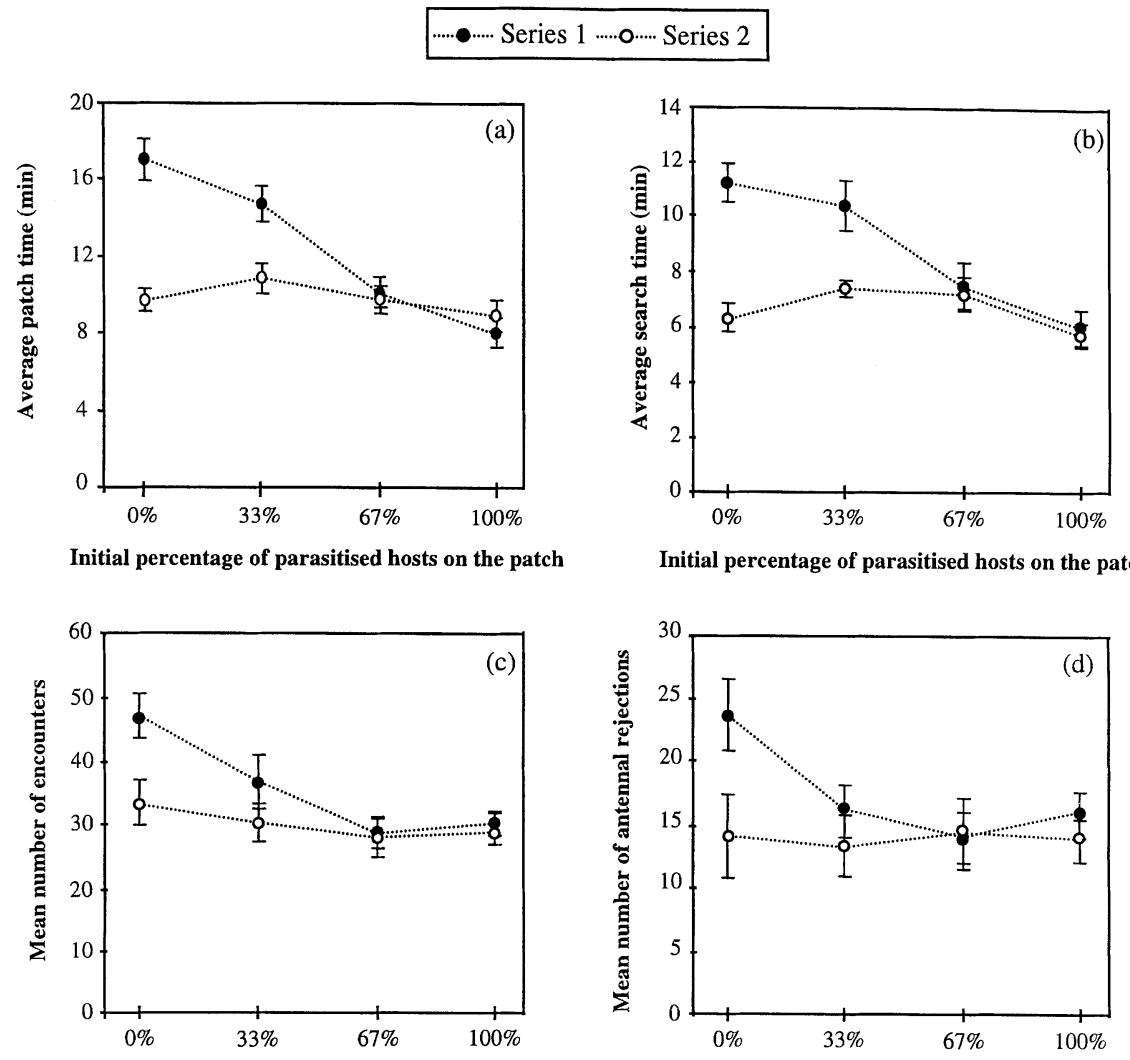

Initial percentage of parasitised hosts on the patch

Initial percentage of parasitised hosts on the patch

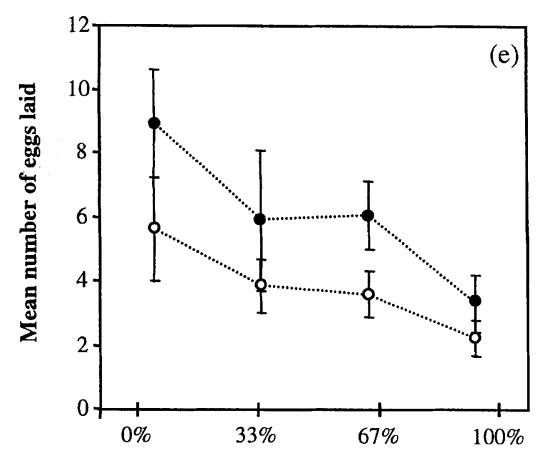

Initial percentage of parasitised hosts on the patch

Fig. 2. Effects of previous experience (series 1 vs. series 2) and patch quality on (a) average total patch time, (b) average searching time within patch, (c) mean number of encounters, (d) mean number of antennal rejections, and (e) mean number of eggs laid of Aphidius rhopalosiphi on patches containing the grain aphid Sitobion avenae as hosts. Error bars indicate SE. Each value is the average of 10 replicates.

and experience of the female also had a significant effect on the time spent searching (Table 1). More particularly, searching time decreased with decreasing patch quality, and females searched for less time in patches after a previous experience (Fig. 2b).

The random effect (the tested female) had a significant effect on the total number of encounters and the number of antennal rejections (Table 1). These results accounted for the great variability between the tested females on these variables.
The two fixed effects (previous experience and patch quality) and their interaction had a significant influence on the total number of host encounters. In series 1 , this number decreased with the proportion of previously parasitised aphids in the patches (Fig. 2c), however in series 2, the mean number of encounters did not seem to vary with patch quality. Finally, the number of antennal rejections displayed by A. rhopalosiphi females was also affected by female experience and patch quality. In the two series, results of this variable were similar 
Table 2. Effects of previous experience (series 1 vs. series 2) and patch quality on the distribution of eggs over unparasitised hosts by Aphidius rhopalosiphi on patches containing the grain aphid Sitobion avenae as host. $\chi^{2}$ tests for random distributions $\left(H_{0}\right.$ : females laid their eggs randomly among initially unparasitised hosts) (see Shu-sheng et al., 1984). All replicates were grouped in each treatment.

\begin{tabular}{|c|c|c|c|c|c|c|c|c|}
\hline \multirow[b]{3}{*}{ Egg(s)/aphid } & \multicolumn{8}{|c|}{ Patch quality } \\
\hline & \multicolumn{2}{|c|}{$\begin{array}{l}12 \text { unparasitised } \\
\text { aphids }\end{array}$} & \multicolumn{2}{|c|}{$\begin{array}{l}8 \text { unparasitised aphids } \\
+4 \text { parasitised aphids }\end{array}$} & \multicolumn{2}{|c|}{$\begin{array}{l}4 \text { unparasitised aphids } \\
+8 \text { parasitised aphids }\end{array}$} & \multicolumn{2}{|c|}{$\begin{array}{l}12 \text { parasitised } \\
\text { aphids }\end{array}$} \\
\hline & Series 1 & Series 2 & Series 1 & Series 2 & Series 1 & Series 2 & Series 1 & Series 2 \\
\hline 0 & 58 & 74 & 52 & 51 & 20 & 22 & - & - \\
\hline 1 & 46 & 38 & 18 & 26 & 13 & 16 & - & - \\
\hline 2 & 9 & 6 & 9 & 2 & 4 & 1 & - & - \\
\hline 3 & 4 & 2 & 0 & 0 & 2 & 1 & - & - \\
\hline 4 & 3 & 0 & 1 & 1 & 1 & 0 & - & - \\
\hline$\chi^{2}$ test & 114.5 & 74.3 & 59.57 & 63.77 & 21.17 & 11 & - & - \\
\hline d.f. $\dagger$ & 110 & 109 & 69 & 70 & 26 & 25 & - & - \\
\hline $\mathrm{p}$ & NS & NS & NS & NS & NS & NS & - & - \\
\hline
\end{tabular}

$\dagger \chi^{2}$ test for random distribution excludes replicates where no eggs were laid by the parasite in unparasitised hosts.

Table 3. Effects of previous experience (series 1 vs. series 2) and patch quality on the distribution of eggs over parasitised hosts by Aphidius rhopalosiphi on patches containing the grain aphid Sitobion avenae as host. All replicates were pooled over each treatment.

\begin{tabular}{|c|c|c|c|c|c|c|c|c|}
\hline \multirow[b]{3}{*}{ Egg(s)/aphid } & \multicolumn{8}{|c|}{ Patch quality } \\
\hline & \multicolumn{2}{|c|}{$\begin{array}{l}12 \text { unparasitised } \\
\text { aphids }\end{array}$} & \multicolumn{2}{|c|}{$\begin{array}{l}8 \text { unparasitised aphids } \\
+4 \text { parasitised aphids }\end{array}$} & \multicolumn{2}{|c|}{$\begin{array}{l}4 \text { unparasitised aphids } \\
+8 \text { parasitised aphids }\end{array}$} & \multicolumn{2}{|c|}{$\begin{array}{l}12 \text { parasitised } \\
\text { aphids }\end{array}$} \\
\hline & Series 1 & Series 2 & Series 1 & Series 2 & Series 1 & Series 2 & Series 1 & Series 2 \\
\hline 0 & - & - & - & - & - & - & - & - \\
\hline 1 & - & - & 24 & 29 & 44 & 56 & 80 & 84 \\
\hline 2 & - & - & 8 & 5 & 20 & 13 & 17 & 20 \\
\hline 3 & - & - & 1 & 0 & 4 & 1 & 3 & 1 \\
\hline 4 & - & - & 2 & 0 & 2 & 0 & 3 & 0 \\
\hline
\end{tabular}

to the results of the total number of encounters (Fig. 2d). Furthermore, it should be noted that even when only unparasitised aphids were on a patch, females rejected many of them after antennation.

Dissection results showed that both patch quality and previous patch experience affected the mean number of eggs laid per female significantly (Table 1). In both series, the number of ovipositions decreased with an increase in the proportion of parasitised aphids in the patch (Fig. 2e). Females laid fewer eggs on the second patches than on the first patches. The distribution of larvae was studied among both unparasitised and previously parasitised aphids. Results for unparasitised aphids in series 1 and series 2 are given in Table 2 . In both series, the initially unparasitised aphids mostly contained zero or one parasitic larva. In the patches with only unparasitised aphids, 48 and $62 \%$ of them escaped from parasitism in series 1 and 2 respectively. Even if all hosts were encountered by parasitoids during the exploitation of a patch, only a few encounters resulted in oviposition. Thus, females rejected some healthy hosts and there was incomplete exploitation of these hosts. The percentage of hosts with no parasitic larva was not affected by patch quality (in series $1: \chi^{2}=5.706$, d.f. $=2$, $P=$ NS; series $2: \chi^{2}=0.877$, d.f. $=2, P=$ NS). In addition, some initially healthy hosts had large numbers of parasitic larvae (up to four) (Table 2). It was shown in the first experiment that females can lay two eggs in an unparasitised host during a single attack. Such behaviour can explain only a part of the rate of self-superparasitism found here because some initially healthy aphids contained more than two parasitic larvae. Thus, the females accepted for oviposition some aphids freshly parasitised by themselves while they rejected other healthy hosts. This suggests that females laid their eggs randomly over initially unparasitised hosts. This assumption was checked using the $\chi^{2}$ statistics described by Shu-sheng etal. (1984), testing whether observed values were distributed randomly (Table 2). In all cases, the distribution of eggs over unparasitised aphids was not distinguishable from the null hypothesis of a random distribution. This suggests that $A$. rhopalosiphi lays its eggs randomly over initially unparasitised hosts, leading to a proportion of self-superparasitism predicted by the Poisson distribution. Finally, female experience had no effect on the number of ovipositions in initially healthy hosts 
in each patch, as no difference was found between series 1 and series $2\left(F_{1,58}=2.71, P=\mathrm{NS}\right)$.

The frequency distributions of larvae among previously parasitised aphids for both series are given in Table 3. These results revealed that only a few eggs were laid in previously parasitised aphids. No significant effect of presence of unparasitised aphids in the patch was found on the frequency distribution of eggs over the parasitised aphids (series 1: $\chi^{2}=4.09$, d.f. $=2, P=\mathrm{NS}$; series $2: \chi^{2}=0.51$, d.f. $=2, P=\mathrm{NS}$ ), however there was a significant effect of previous experience on the number of ovipositions in already parasitised hosts of each patch $\left(F_{1,58}=6.71, P<0.05\right)$. Experienced females laid fewer eggs in parasitised aphids than did inexperienced females.

\section{Discussion}

\section{Host discrimination in Aphidius rhopalosiphi}

These results show that when females have to choose between healthy hosts and aphids parasitised $3 \mathrm{~h}$ earlier, they tend to avoid laying eggs in previously parasitised hosts. Thus, A. rhopalosiphi may be able to recognise previously parasitised hosts. Some cases of superparasitism were observed, however, in particular when females faced only initially unparasitised hosts in the second experiment. A random distribution of eggs among initially healthy hosts was observed, indicating that females may accept freshly parasitised aphids. Two hypotheses may explain such self-superparasitism: either females are able to recognise freshly parasitised hosts but decide to superparasitise them anyway or such hosts simply cannot be discriminated. Supposing females decide to superparasitise hosts, this is related to the fact that self-superparasitism could be adaptive under certain conditions. Visser et al. (1992) showed with an evolutionarily stable strategy model that superparasitism is an adaptive strategy for a solitary parasitoid depleting a patch with a number of competing conspecifics. In the present study, females searched alone in patches (i.e. without direct competition with conspecifics) and under such a situation, self-superparasitism is always considered to be maladaptive (Visser etal., 1990). Despite this, there were many cases of self-superparasitism whereas many healthy hosts were still available. Thus, in the present case, self-superparasitism might rather be related to a complete lack of host discrimination on freshly parasitised hosts, and such a hypothesis would imply a random distribution of eggs over initially healthy hosts. Consequently, because A. rhopalosiphi was able to recognise hosts parasitised $3 \mathrm{~h}$ earlier but not hosts just parasitised, host discrimination in this aphid parasitoid would appear to require a few hours to become effective.

This temporal pattern suggests that host discrimination in $A$. rhopalosiphi is based on a marking cue that can be detected only a few hours after parasitism. During this time interval, host discrimination would be ineffective. The relatively long time (a few hours) that must elapse before any evidence of discrimination suggests that parasitoid-injected or deposited substances are unlikely to be involved; however a few hours would probably be enough to induce changes in the aphid to become detectable. Various chemical changes are known to occur in the haemolymph of parasitised hosts (e.g. Pennachio et al., 1995; Bischof \& Ortel, 1996). It has been suggested that such chemical changes might provide the basis of host discrimination (Fisher \& Ganesalingam, 1970; Vinson, 1976; Cloutier etal., 1984).

Even if females are able to recognise hosts parasitised $3 \mathrm{~h}$ earlier, some initially parasitised aphids were still attacked. These levels of conspecific-superparasitism could be the result of either females' decision to superparasitise or imperfect host discrimination: $3 \mathrm{~h}$ would be enough for inducing chemical changes but these changes would not be strong enough to lead to perfect discrimination.

Does an ovipositional experience improve the ability to discriminate between unparasitised and parasitised hosts? Dynamic optimal diet models predict that parasitised hosts are more likely to be rejected than healthy hosts, and that inexperienced females are more likely to accept parasitised hosts than are experienced females (Colazza et al., 1996). The results presented here are partially in agreement with these predictions. The parasitised hosts are less accepted than unparasitised hosts but ovipositional experience does not influence host acceptance decisions. The fact that inexperienced females also rejected hosts parasitised $3 \mathrm{~h}$ earlier, suggests that learning was not involved in host discrimination.

\section{Host discrimination and patch exploitation strategy}

Total patch time and searching time decreased when the proportion of parasitised aphids increased on a patch of constant size. This result is probably related to the female's perception of the potential profitability of the patch. Therefore, the higher the expectation of A. rhopalosiphi encountering unparasitised hosts, the longer the time a female should invest in the patch. Conversely, if a female perceived that the probability of encountering unparasitised hosts is low, it would reduce the time spent on the patch and would move on and search for a better patch. These results are in agreement with other studies that have found an incremental effect of encounters with parasitised hosts on the tendency to leave (see Wajnberg et al., 1999 for a review). Host discrimination due to recognition of previously parasitised aphids may thus allow females to adjust their patch residence time in response to the level of profitability of the patch.

The effect of patch quality on patch residence time is a classical result, however dissections of hosts revealed an unusual ovipositional behaviour: even if all the initially healthy hosts were handled by females during patch depletion, only a few encounters resulted in parasitism. Several hypotheses could explain the partial resource exploitation by parasitoids (see Rosenheim \& Mangel, 1994); the most wellknown is the marginal value theorem developed by Charnov (1976). According to this model, each patch should be exploited optimally until the encounter rate with unparasitised aphids drops below a marginal value that is the mean rate of fitness gain that can be achieved in all the available patches in 
the environment. Parasitoids should then leave before a patch is fully exploited. Thus, according to Charnov's theorem, the patch leaving decision is based on the encounter rate with healthy hosts and its estimate depends strongly on the ability of the female to discriminate between unparasitised hosts and hosts already parasitised. Such an ability was not found in $A$. rhopalosiphi so Charnov's theorem is unlikely to apply in the present case. In fact, imperfect host discrimination presents the problem to the wasp of decision-making with partial information. This partial lack of host discrimination is probably the main factor that explains the partial exploitation of unparasitised hosts in A. rhopalosiphi. Because encounters with hosts are likely to be random events and because females are probably unable to recognise freshly parasitised hosts, females may experience a risk of self-superparasitism. In such cases, in order to avoid self-superparasitism, an optimal strategy would be early patch departure after ovipositing in only a few unparasitised hosts (Rosenheim \& Mangel, 1994). The observed level of healthy aphid exploitation indicates that such a strategy could very well be adopted by A. rhopalosiphi.

\section{Previous experience and patch exploitation strategy}

Despite ovipositional experience having no effect on discrimination ability in A. rhopalosiphi, there is a question of whether experience on a previous patch affects subsequent patch exploitation strategy. Waage (1979) studied the effect of a patch visit by $V$. canescens on the residence time on a subsequent patch. When host densities of both patches were the same, the visit to the second patch was shorter. Waage (1979) supposed that this result might be caused by habituation to concentrations of specific kairomones. In the present study, the patch exploitation behaviour of two identical patches was compared. Results indicated that on high-quality patches, the same female spends less time on the second patch than on the first patch, and the distribution of eggs over unparasitised aphids does not differ between the two patches. Such results suggest that females exploit the second patch in the same way in a shorter time. Hence, experience can apparently modify the female's original estimate of patch quality. Aphidius rhopalosiphi uses the information gained from past experiences with the same patch quality to reduce the time and energy invested in a patch quality estimate. Such a mechanism may be considered to be adaptive as it allows a time saving.

In low-quality patches, previous experience did not affect total patch time. Whatever their experience, females must always invest the same time for the recognition of a lowquality patch. In addition, a previous experience affects the egg distribution in already parasitised hosts. Females lay fewer eggs in these aphids on the second patch. Thus, a previous experience can be adaptive on low-quality patches because it decreases the wastage of eggs.

Gardner etal. (1984) found that A. rhopalosiphi did not discriminate between unparasitised and freshly parasitised aphids. The present results are in partial agreement with these authors because a lack of host discrimination on freshly parasitised hosts was indeed found, but females were able to recognise hosts that were parasitised $3 \mathrm{~h}$ earlier. Thus, host discrimination was shown in A. rhopalosiphi but this capacity would probably need a few hours before being effective. Moreover, the present study revealed that A. rhopalosiphi exploits host patches incompletely. This has been demonstrated for a few other parasitoids (e.g. Cronin \& Strong, 1993; Mackauer \& Völkl, 1993) and several hypotheses have been proposed to explain the partial exploitation of patches. The experimental approach presented seems to support the model developed by Rosenheim and Mangel (1994), which assumed that imperfect host discrimination could promote earlier patch leaving. In addition, females can save time and eggs by using their experience, because previous experience on a patch of the same quality induces lower patch time allocation and lower conspecific-superparasitism. Thus, even if host discrimination is imperfect, experience may lead the female to forage in a different, more optimal way. These results suggest new experiments to investigate the role of experience in more realistic environments in which patches of different quality would be offered to the females. Finally, such an approach could be applicable to the selection of natural enemies for biological control programmes. Parasitoids that demonstrate incomplete exploitation of host patches as an optimal strategy would probably be less efficient control agents than other species.

\section{Acknowledgements}

We are grateful to M. Hochberg (University of Montpellier II), M. Plantegenest (E.N.S.A. Rennes), J.C. Simon (I.N.R.A. Rennes) and two anonymous referees for their useful comments and suggestions on an earlier draft of the manuscript. We thank also F. Lefevre and B. Chaubet for their technical assistance. This study was supported by grants from the Ministère de l'Agriculture and from the Ministère de l'Education, de la Recherche et de la Technologie, France.

\section{References}

van Alphen, J.J.M. (1993) Patch residence time and encounters with parasitised hosts: a reaction. Netherlands Journal of Zoology, 43, 340-349.

van Alphen, J.J.M. \& Galis, F. (1983) Patch time allocation and parasitization efficiency of Asobara tabida, a larval parasitoid of Drosophila. Journal of Animal Ecology, 52, 937-952.

van Alphen, J.J.M. \& Vet, L.E.M. (1986) An evolutionary approach to host finding and selection. Insect Parasitoids (ed. by J. K. Waage and D. Greathead), pp. 23-61. Academic Press, London.

Bischof, C. \& Ortel, J. (1996) The effects of parasitism by Glytapanteles liparidis (Braconidae: Hymenoptera) on the hemolymph and total body composition of gypsy moth larvae (Lymantria dispar, Lymantriidae: Lepidoptera). Parasitology Research, 82, 687-692.

Charnov, E.L. (1976) Optimal foraging: the marginal value theorem. Theoretical Population Biology, 9, 129-136.

Cloutier, C., Dohse, L.A. \& Bauduin, F. (1984) Host discrimination in the aphid parasitoid Aphidius nigripes. Canadian Journal of Zoology, 62, 1367-1372. 
Colazza, S., Rosi, M.C., Sebastiani, P. \& Ursini, M. (1996) Host acceptance behaviour in the egg parasitoid Trissolcus basalis (Hymenoptera: Scelionidae). Acta Oecologia, 17, 109-125.

Collins, M.D. \& Dixon, A.F.G. (1986) The effect of egg depletion on the foraging behaviour of an aphid parasitoid. Journal of Applied Entomology, 102, 342-352.

Cronin, J.T. \& Strong, D.R. (1993) Substantially submaximal oviposition rates by a mymarid egg parasitoid in the laboratory and field. Ecology, 74, 1813-1825.

Driessen, G., Bernstein, C., van Alphen, J.J.M. \& Kacelnik, A. (1995) A count-down mechanism for host search in the parasitoid Venturia canescens. Journal of Animal Ecology, 64, 117-125.

Fisher, R.C. \& Ganesalingam, V.K. (1970) Changes in the composition of host haemolymph after attack by an insect parasitoid. Nature, 222, 191-192.

Gardner, S.M., Ward, S.A. \& Dixon, A.F.G. (1984) Limitation of superparasitism by Aphidius rhopalosiphi: a consequence of aphid defensive behaviour. Ecological Entomology, 9, 149-155.

Hemerik, L., Driessen, G. \& Haccou, P. (1993) Effects of intra-patch experiences on patch time, search time and searching efficiency of the parasitoid Leptopilina clavipes. Journal of Animal Ecology, 62, 33-44.

Iwasa, Y., Higashi, M. \& Yamamura, N. (1981) Prey distribution as a factor determining the choice of optimal foraging strategy. American Naturalist, 117, 710-723.

Krespi, L., Dedryver, C.A., Rabasse, J.M. \& Nenon, J.P. (1994) A morphometric comparison of aphid mummies containing diapausing vs non-diapausing larvae of Aphidius rhopalosiphi (Hymenoptera: Braconidae, Aphidiinae). Bulletin of Entomological Research, 84, $45-50$

van Lenteren, J.C. (1981) Host discrimination by parasitoids. Semiochemicals, their Role in Pest Control (ed. by D.A. Nordlund, R. L. Jones and W. J. Lewis), pp. 153-179. WileyInterscience, New York.

Mackauer, M. (1972) Antennal amputation as a method for biomarking aphids. Journal of Economic Entomology, 65, 1725-1727.

Mackauer, M. \& Völkl, W. (1993) Regulation of aphid populations by aphidiid wasps: does parasitoid foraging behaviour or hyperparasitism limit impact? Oecologia, 94, 339-350.

McCullagh, P. \& Nelder, J.A. (1989) Generalized Linear Models, 2nd edn. Chapman \& Hall, New York.

McNamara, J. \& Houston, A. (1980) The application of statistical decision theory to animal behaviour. Journal of Theoretical Biology, 85, 673-690.

McNamara, J. \& Houston, A. (1985) A simple model of information use in the exploitation of patchily distributed food. Animal Behavior, 33, 553-560.

Papaj, D.R., Snellen, H., Swaans, K. \& Vet, L.E.M. (1994) Unrewarding experiences and their effect on foraging in the parasitic wasp Leptopilina heterotoma (Hymenoptera: Eucoilidae). Journal of Insect Behavior, 7, 465-481.
Pennachio, F., Digilio, M.C. \& Tremblay, E. (1995) Biochemical and metabolic alterations in Acyrthosiphon pisum parasitised by Aphidius ervi. Archives of Insect Biochemistry and Physiology, 30, 351-367.

Rosenheim, J.A. \& Mangel, M. (1994) Patch-leaving rules for parasitoids with imperfect host discrimination. Ecological Entomology, 19, 374-380.

Rosenheim, J.A. \& Rosen, D. (1991) Foraging and oviposition decisions in the parasitoid Aphytis lingnanensis: distinguishing the influences of egg load and experience. Journal of Animal Ecology, 60, 873-893.

Shu-sheng, L., Morton, R. \& Hughes, R.D. (1984) Oviposition preferences of a hymenopterous parasite for certain instars of its aphid host. Entomologia experimentalis et applicata, 35, 249-254.

van Steenis, M.J., El-Khawass, K.A.M.H., Hemerik, L. \& van Lenteren, J.P. (1996) Time allocation of the parasitoid Aphidius colemani (Hymenoptera: Aphidiidae) foraging for Aphis gossypii (Homoptera: Aphidae) on cucumber leaves. Journal of Insect Behavior, 9, 283-295.

Stephens, D.W. (1990) Risk and incomplete information in behavioral ecology. Risk and Uncertainty in Tribal and Peasant Economies (ed. by E. Cashdan), pp. 19-46. Westview Press, Boulder, Colorado.

Vet, L.E.M., Lewis, W.J. \& Cardé, R.T. (1995) Parasitoid foraging and learning. Chemical Ecology of Insects, 2nd edn (ed. by W. Bell and R. T. Cardé), pp. 65-101. Chapman \& Hall, London.

Vet, L.E.M., Lewis, W.J., Papaj, D.R. \& van Lenteren, J.C. (1990) A variable response model for parasitoid foraging behavior. Journal of Insect Behavior, 3, 471-490.

Vinson, S.B. (1976) Host selection by insect parasitoids. Annual Review of Entomology, 21, 109-113.

Visser, M.E., van Alphen, J.J.M. \& Hemerik, L. (1992) Adaptive superparasitism and patch time allocation in solitary parasitoids: an ESS model. Journal of Animal Ecology, 61, 93-101.

Visser, M.E., van Alphen, J.J.M. \& Nell, H.W. (1990) Adaptive superparasitism and patch time allocation in solitary parasitoids: the influence of the number of parasitoids depleting a patch. Behaviour, 114, 21-36.

Vos, M., Hemerik, L. \& Vet, L.E.M. (1998) Patch exploitation by the parasitoids Cotesia rubecula and Cotesia glomerata in multi-patch environments with different host distributions. Journal of Animal Ecology, 67, 774-783.

Waage, J.K. (1979) Foraging for patchily-distributed hosts by the parasitoid, Nemertis canescens. Journal of Animal Ecology, 48, 353-371.

Wajnberg, E., Rosi, M.C. \& Colazza, S. (1999) Genetic variation in patch time allocation in a parasitic wasp. Journal of Animal Ecology, 68, 121-133.

Accepted 1 August 2000 